\title{
Lysine-specific demethylase 1 promotes brown adipose tissue thermogenesis via repressing glucocorticoid activation
}

\author{
Xing Zeng, ${ }^{1,2}$ Mark P. Jedrychowski, ${ }^{2}$ Yi Chen, ${ }^{1,2}$ Sara Serag, ${ }^{1}$ Gareth G. Lavery, ${ }^{3}$ Steve P. Gygi, ${ }^{2}$ \\ and Bruce M. Spiegelman ${ }^{1,2}$ \\ ${ }^{1}$ Dana-Farber Cancer Institute, Boston, Massachusetts, 02115, USA; ${ }^{2}$ Department of Cell Biology, Harvard Medical School, \\ Boston, Massachusetts, 02115, USA; ${ }^{3}$ Institute of Metabolism and Systems Research, University of Birmingham, Birmingham \\ B15 2TT, United Kingdom
}

Brown adipocytes display phenotypic plasticity, as they can switch between the active states of fatty acid oxidation and energy dissipation versus a more dormant state. Cold exposure or $\beta$-adrenergic stimulation favors the active thermogenic state, whereas sympathetic denervation or glucocorticoid administration promotes more lipid accumulation. Our understanding of the molecular mechanisms underlying these switches is incomplete. Here we found that LSD1 (lysine-specific demethylase 1), a histone demethylase, regulates brown adipocyte metabolism in two ways. On the one hand, LSD1 associates with PRDM16 to repress expression of white fat-selective genes. On the other hand, LSD1 represses HSD11B1 (hydroxysteroid 11- $\beta$-dehydrogenase isozyme 1), a key glucocorticoid-activating enzyme, independently from PRDM16. Adipose-specific ablation of LSD1 impaired mitochondrial fatty acid oxidation capacity of the brown adipose tissue, reduced whole-body energy expenditure, and increased fat deposition, which can be significantly alleviated by simultaneously deleting HSD11B1. These findings establish a novel regulatory pathway connecting histone modification and hormone activation with mitochondrial oxidative capacity and whole-body energy homeostasis.

[Keywords: LSD1; PRDM16; HSD11B1; brown adipose tissue; thermogenesis; mitochondria]

Supplemental material is available for this article.

Received June 9, 2016; revised version accepted July 28, 2016.

The increased prevalence of obesity poses a significant challenge to human health, as obese individuals are predisposed to multiple pathological conditions, such as cardiovascular diseases, type 2 diabetes, hypertension, and certain types of cancer (Després and Lemieux 2006; Gaal et al. 2006; Kahn et al. 2006; Basen-Engquist and Chang 2011). Obesity or excessive expansion of the adipose tissue is a consequence of chronic energy imbalance between energy intake and dissipation. Two major classes of adipose tissues exist in mammals-white and brown -with distinct anatomical locations, developmental origins, and physiological functions. White adipose tissue (WAT) has low mitochondrial content and is specialized for storing excess energy in the form of triacylglycerides. In contrast, brown adipose tissue (BAT) features very high mitochondrial content and is capable of performing high rates of fatty acid oxidation (FAO) followed by dissipation of the harnessed energy in the form of heat, a process known as nonshivering thermogenesis (Cannon and Nedergaard 2004). Promoting FAO and thermogenesis

Corresponding author: bruce_spiegelman@dfci.harvard.edu Article published online ahead of print. Article and publication date are online at http://www.genesdev.org/cgi/doi/10.1101/gad.285312.116. represents a potential therapy to increase energy expenditure and combat obesity.

Both the BAT and WAT display phenotypic plasticity, as each type can take on the counterpart's morphological and functional features in response to temperature or hormone stimuli. Under conditions such as cold exposure or $\beta$-adrenergic stimulation, cells similar to brown adipocytes emerge in the WAT depots (Loncar 1991; Ghorbani and Himms-Hagen 1997), an inducible population also known as the "beige" adipocytes. Beige cells are a distinct cell type from classical brown fat cells and have different developmental origins (Wu et al. 2012; Long et al. 2014). It is important to note that sympathetic denervation or glucocorticoid (GC) administration represses the FAO/thermogenic capacity of the BAT and causes it to enter a dormant state. Such effects are fully reversible upon $\beta$-adrenergic agonist supplementation or GC retrieval (Dulloo and Miller 1984; Minokoshi et al. 1986; Strack et al. 1995).

(C) 2016 Zeng et al. This article is distributed exclusively by Cold Spring Harbor Laboratory Press for the first six months after the full-issue publication date (see http://genesdev.cshlp.org/site/misc/terms.xhtml). After six months, it is available under a Creative Commons License (Attribution-NonCommercial 4.0 International), as described at http:// creativecommons.org/licenses/by-nc/4.0/. 
However, the molecular basis of the phenotypic plasticity of adipocytes remains to be fully explored.

Our laboratory previously identified a BAT-enriched transcriptional coregulator, PRDM16, that drives the expression of mitochondrial and thermogenic genes in brown and beige adipocytes (Seale et al. 2007). Transgenic expression of PRDM16 in the adipose tissues results in increased levels of mitochondrial and thermogenic genes in the WAT, reflecting expansion of the beige population (Seale et al. 2011). Conversely, ablation of PRDM16 markedly inhibits beige adipocyte development and function (Cohen et al. 2014). Classical brown fat required deletion of both PRDM16 and PRDM3 to ablate thermogenic gene expression completely, but BAT from mice deleted in only PRDM16 show marked age-related reduction in expression of a wide range of mitochondrial and thermogenic genes (Harms et al. 2014). These findings have clearly established a role of PRDM16 in driving the thermogenic program in both brown and beige adipocytes.

Whereas PRDM16's function in the transcriptional activation of the thermogenic gene program has been well established, two lines of evidence suggest that it could also act as a transcriptional corepressor. First, knocking down PRDM16 from the precursors isolated from the BAT results in differentiation of these cells into myocytes (Seale et al. 2008). Interestingly, lineage tracing experiments revealed that both the BAT and skeletal muscle are derived from a common population of myf5-positive progenitors (Seale et al. 2008). These observations therefore suggest that PRDM16 may repress the myogenic program during the process of brown adipocyte differentiation. Second, ablating PRDM16 from the BAT results in increased expression of WAT-selective genes (Harms et al. 2014). Importantly, the up-regulation of WAT-selective genes is evident in the BAT of mice lacking PRDM16 as young as $6 \mathrm{wk}$, whereas significant down-regulation of thermogenic genes does not occur until the mice reach 3-6 mo of age (Harms et al. 2014). These observations suggest that the increased expression of WAT-selective genes is probably not secondary to the decreased expression of the BAT-selective thermogenic genes; instead, it may reflect a primary consequence of loss of transcriptional repression by PRDM16. Consistent with this, PRDM16 was found to physically associate with the transcriptional corepressors CTBP1 and CTBP2, and its ability to repress some WAT-selective genes in adipocytes is dependent on these corepressors (Kajimura et al. 2008). Given the evidence that PRDM16 represses both the myogenic and WAT-selective genes in brown adipocytes, the molecular mechanism underlying PRDM16-mediated transcriptional repression becomes an important question to address.

Here, we show that PRDM16 interacts with lysine-specific demethylase 1 (LSD1, also known as KDM1A), a repressive histone-modifying enzyme that demethylates monomethyl-H3K4 or dimethyl-H3K4, both of which are histone markers closely associated with promoters and enhancers of actively transcribed genes. LSD1 deficiency impairs the FAO/thermogenic capacity of the BAT and reduces whole-body energy expenditure; this could be substantially alleviated by simultaneously deleting HSD11B1 (hydroxysteroid 11- $\beta$-dehydrogenase isozyme 1), a gene controlling GC activation that is induced by LSD1 deficiency. Taken together, these results establish that LSD1 plays a critical role in BAT via repressing HSD11B1, thus preventing local GC excess.

\section{Results}

\section{LSD1 associates with PRDM16 and represses WAT-selective genes in brown adipocytes}

To understand the molecular basis of PRDM16-mediated repression of WAT-selective genes in BAT, we immunepurified the endogenous PRDM16 complex from nuclear extracts of primary brown adipocytes with antibody highly specific to PRDM16 (Supplemental Fig. S1). Bound proteins were eluted with glycine and subjected to quantitative mass spectrometry with isobaric tagging. A series of known members of the CTBP corepressor complex were found to be highly enriched in the anti-PRDM16 pulldown relative to the IgG control, including CTBP1, CTBP2, RCOR1, RCOR3, ZNF516, HDAC1, HDAC2, and KDM1A (Table 1; Shi et al. 2003). The same set of proteins was identified when we purified Flag-tagged PRDM16 expressed in primary brown adipocytes via adenoviral infection (Table 2). A complete list of the peptides identified is in Supplemental Table S1. We confirmed the presence of KDM1A and CTBP1 in the endogenous or Flag-tagged PRDM16 complex by Western blot (Fig. 1A).

KDM1A, commonly known as LSD1, demethylates monomethylated or dimethylated histone H3 Lys4 (H3K4me1 or H3K4me2), which are histone markers closely associated with active transcription (Shi et al. 2004). H3K4mel is particularly enriched at active enhancers, whereas $\mathrm{H} 3 \mathrm{~K} 4 \mathrm{me} 2$ is more associated with promoter regions of active or poised genes. By removing these modifications, LSD1 acts as a corepressor on its target genes. We therefore investigated whether LSD1 cooperates with PRDM16 to repress WAT-selective genes in the BAT. We treated primary brown adipocytes with S2101, a mechanism-based small molecule inhibitor of LSD1, and observed dose-dependent increases in a series of WAT-selective genes. For example, retn and agt mRNA levels increase by twofold at the highest dose (Fig. 1B).

Table 1. Endogenous PRDM16 is associated with components of the CTBP corepressor complex as identified by mass spectrometry

\begin{tabular}{lcccc}
\hline Gene symbol & Peptide & IgG & PRDM16 & PRDM16/IgG \\
\hline Prdm16 & 60 & 4138.9 & 146060.0 & 35.29 \\
Ctbp1 & 16 & 1992.7 & 47328.3 & 23.75 \\
Znf516 & 38 & 5164.6 & 60967.2 & 11.80 \\
Kdm1a & 19 & 3273.8 & 30511.2 & 9.32 \\
Rcor1 & 8 & 963.0 & 8499.7 & 8.83 \\
Ctbp2 & 5 & 1140.4 & 6262.3 & 5.49 \\
Hdac1 & 10 & 2382.2 & 8779.98 & 3.69 \\
Hdac2 & 4 & 1032.87 & 3526.17 & 3.41 \\
Rcor3 & 4 & 1479.04 & 4535.16 & 3.07 \\
\hline
\end{tabular}


Table 2. Flag-tagged PRDM16 is associated with components of the CTBP corepressor complex as identified by mass spectrometry

\begin{tabular}{lrrrr}
\hline Gene Symbol & Peptides & \multicolumn{1}{c}{ Ad } & \multicolumn{1}{c}{+ Ad } & + Ad/ $-\mathrm{Ad}$ \\
\hline Prdm16 & 103 & 927.6 & 42083.6 & 45.37 \\
Znf516 & 14 & 139.6 & 5561.4 & 39.83 \\
Ctbp2 & 6 & 57.8 & 1282.8 & 22.21 \\
Ctbp1 & 6 & 95.8 & 1752.1 & 18.28 \\
Rcor1 & 6 & 142.1 & 1099.5 & 7.74 \\
Rcor3 & 3 & 111.5 & 806.9 & 7.24 \\
Kdm1a & 5 & 388.4 & 2673.9 & 6.88 \\
Hdac2 & 2 & 209.2 & 549.5 & 2.63 \\
Hdac1 & 3 & 223.4 & 339.0 & 1.52 \\
\hline
\end{tabular}

(Ad) Adenovirus.

These genes are also up-regulated in the PRDM16-deficient BAT (Matthew et al. 2014). Pharmacological inhibition of LSD1 had no effect on the expression of panadipocyte genes, including fabp4, adipoq, and pparg (Fig. 1B). Modest decreases in the expression were also observed for certain BAT-selective genes, such as ucp 1 and cidea (Fig. 1B).
Ablation of LSD1 results in increases in expression of WAT-selective genes in the BAT

We next sought to investigate this phenomenon with a loss-of-function genetic model of LSD1. For this purpose, we generated mice lacking LSD1 specifically in the adipose tissue by crossing an adiponectin-Cre line to a LSD1 floxed line (Kerenyi et al. 2013). The resulting $1 s d 1^{\operatorname{lox} P / \operatorname{lox} P}, \mathrm{cre}^{+}$mice (referred to here as adipose-specific LSD1 knockout [AdLKO]) display a large reduction in both the protein and the mRNA levels of LSD1 in the interscapular BAT, inguinal subcutaneous WAT, and epididymal WAT; such reductions were not observed in the liver (Fig. 2A). We isolated the stromal vascular fractions from the BAT of the control and AdLKO mice and differentiated them into brown adipocytes in vitro. We observed increased expression of the same set of WAT-selective genes in the LSD1-deficient brown adipocytes as was seen with the pharmacological LSD1 inhibition. For example, retn expression increased by 1.8 -fold, and agt increased by 1.5-fold (Fig. 2B). Again, we observed no difference in the expression of pan-adipocyte genes such as fabp4, adipoq, and pparg and only modest decreases in some but not all BAT-selective genes in the LSD1-deficient brown
A

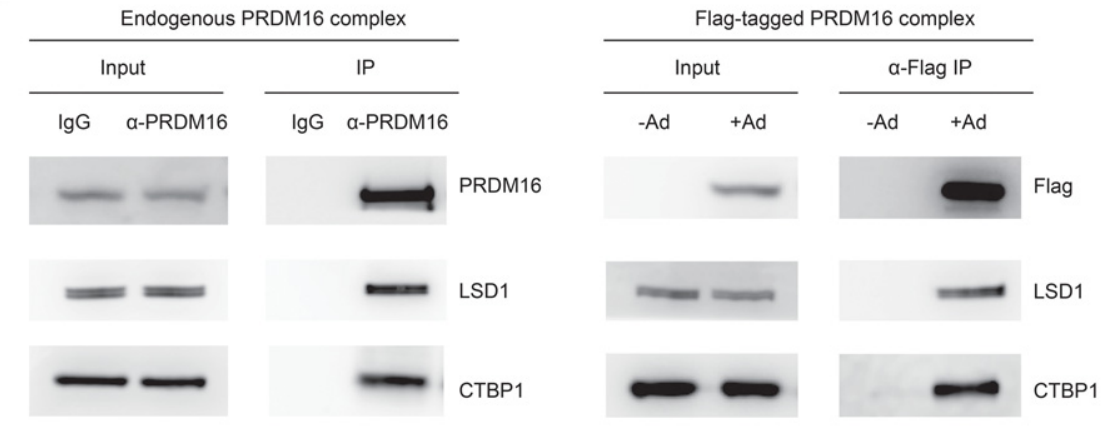

B

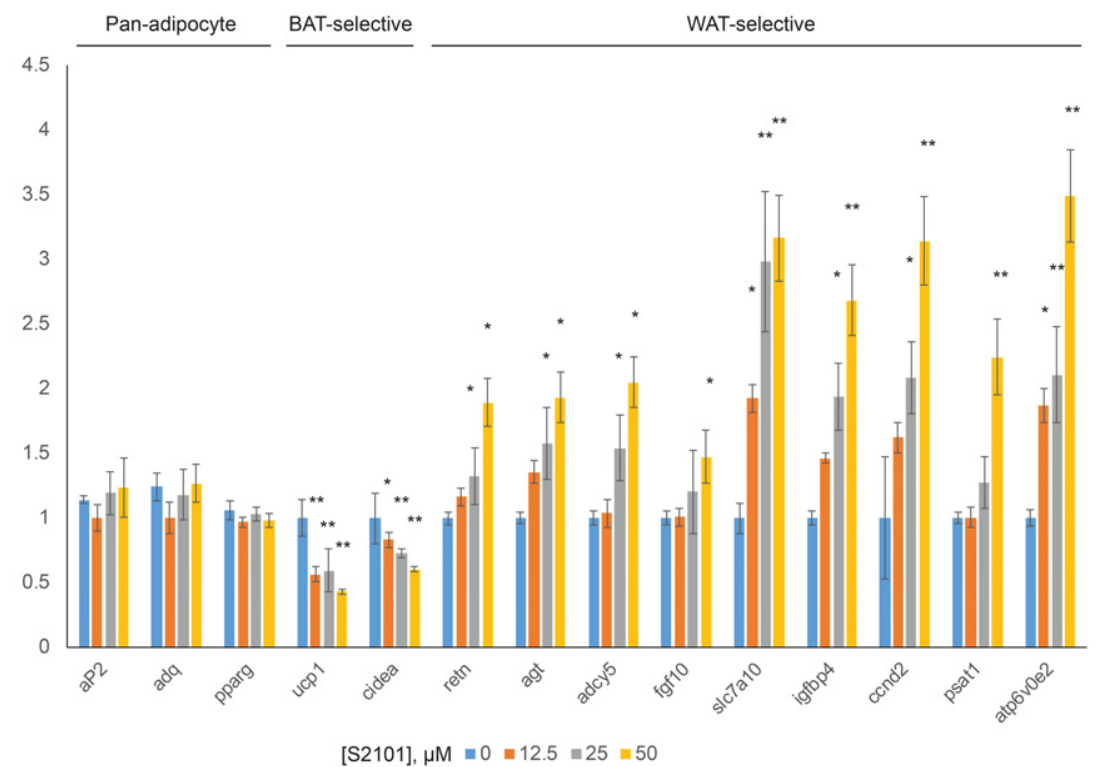

Figure 1. LSD1 associates with PRDM16 and represses white selective genes in brown adipocytes. (A) Western blot for PRDM16, LSD1, and CTBP1 protein levels in purified endogenous or Flag-tagged PRDM16 complexes. (B) mRNA levels of pan-adipocyte and brown- and white-selective genes in brown adipocytes treated with S2101, a small molecule inhibitor of LSD1. Mean \pm SEM. $n=3 .\left(^{*}\right) P<0.05 ;\left({ }^{* *}\right) P<0.01$. 
A

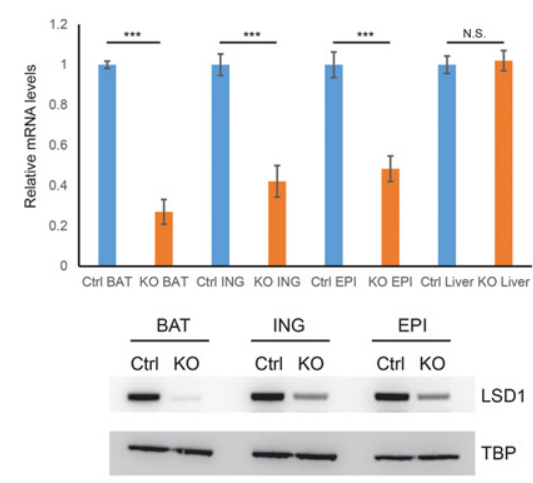

B

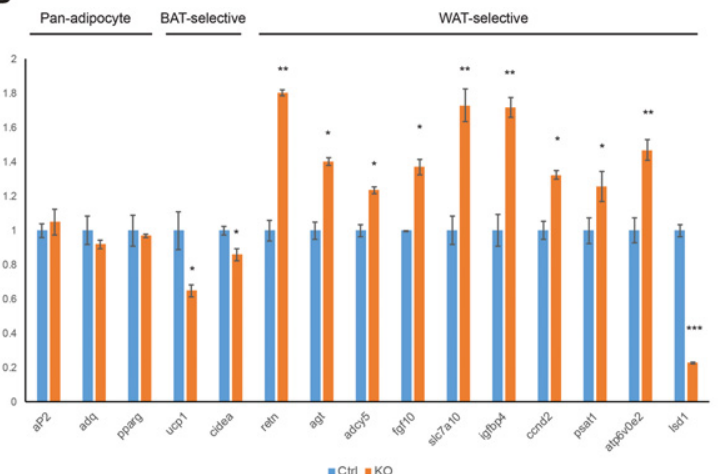

C

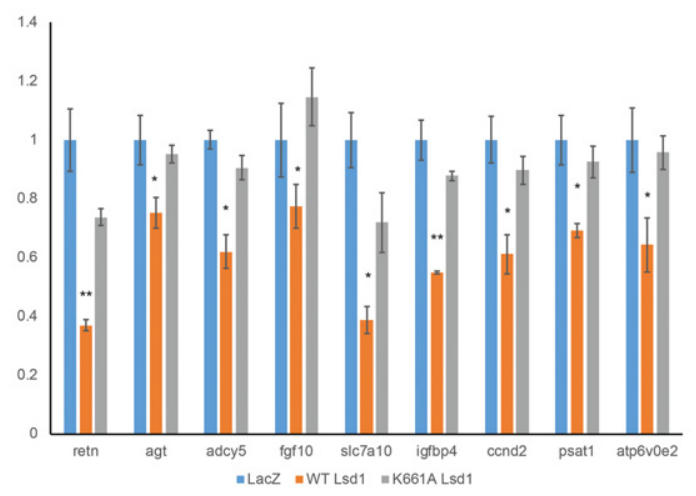

D

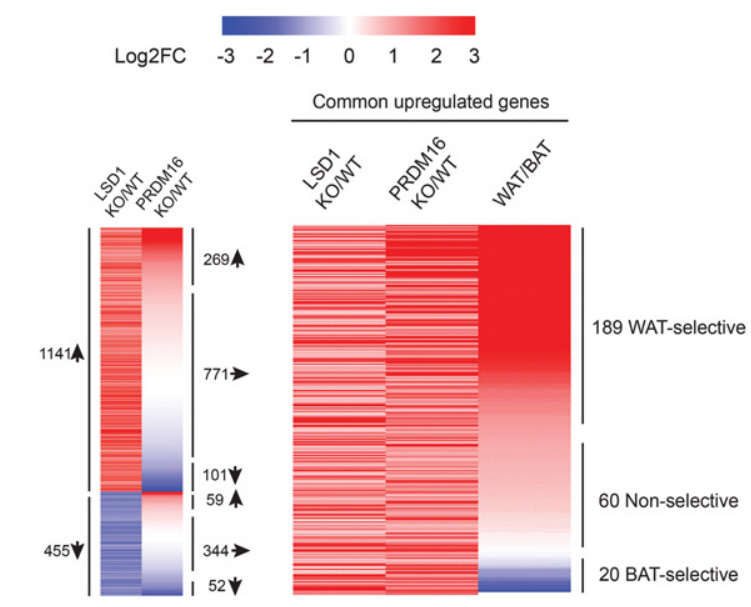

E

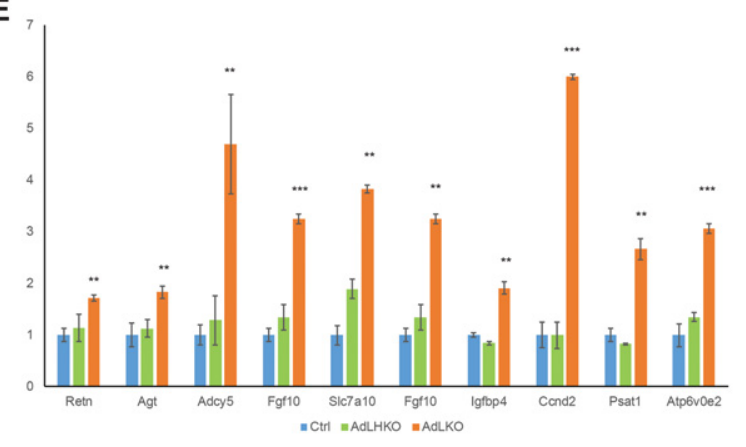

$\mathbf{F}$

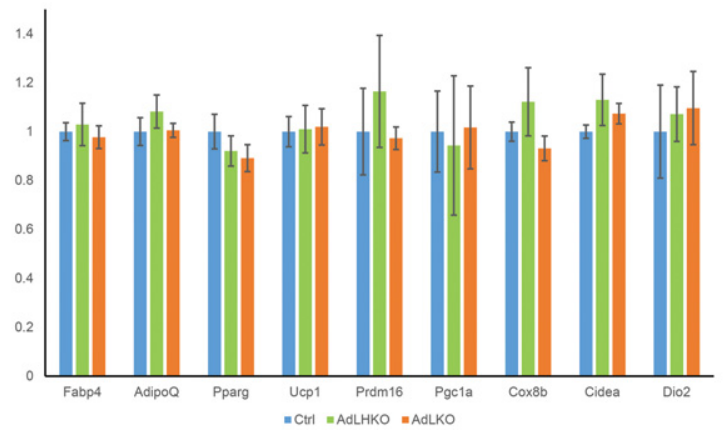

Figure 2. Ablation of LSD1 results in up-regulation of white-selective genes in brown adipocytes. $(A)$ mRNA and protein levels of LSD1 in the adipose tissue of control and AdLKO mice. Mean \pm SEM. $n=5 .\left(^{* * *}\right) P<0.001$. $(B)$ mRNA levels of pan-adipocyte and brown- and whiteselective genes in control and LSD1-deficient brown adipocytes differentiated in vitro. Mean \pm SEM. $n=6 .\left({ }^{*}\right) P<0.05 ;\left(^{* *}\right) P<0.01$. $(C)$ mRNA levels of white-selective genes in LSD1-deficient brown adipocytes expressing wild-type LSD1or a catalytically inactive mutant of LSD1 (K661A). Mean \pm SEM. $n=6 .\left(^{*}\right) P<0.05 ;\left({ }^{*}\right) P<0.01$. $(D)$ Heat map comparison of genes that are significantly changed in the LSD1deficient and PRDM16-deficient BAT relative to the control. $(E)$ mRNA levels of white-selective genes in the control and LSD1-deficient BAT. (AdLHKO) Heterozygous LSD1 knockout. Mean \pm SEM. $\left.n=5 .\left({ }^{* *}\right) P<0.01 ;{ }^{* * *}\right) P<0.001$. $(F)$ mRNA levels of pan-adipocyte and brown-selective genes in the control and LSD1-deficient BAT. (AdLHKO) Heterozygous LSD1 knockout. Mean \pm SEM. $n=5$.

adipocytes relative to the control (Fig. 2B). This is in striking contrast to PRDM16-deficient brown adipocytes in that the latter display strong reduction in the expression of BAT-selective genes (Harms et al. 2014). These observations suggest that the ability of PRDM16 to repress WATselective genes via LSD1 is functionally separable from its ability to activate BAT-selective genes. Importantly, ex- pressing the wild-type LSD1 but not a catalytically inactive mutant of LSD1 (K661A) in the LSD1-deficient brown adipocytes restored repression of the WAT-selective genes (Fig. 2C), indicating that the enzymatic activity of LSD1 is required for repressing the WAT-selective genes.

We next performed a global comparison of the expression profile of the PRDM16-deficient and the LSD1- 
deficient BAT. At a threshold of 1.5-fold change, RNA sequencing (RNA-seq) analysis identified 1141 significantly up-regulated genes and 455 significantly down-regulated genes in the AdLKO relative to the control. A previously published microarray analysis of the PRDM16-deficient BAT identified 1702 significantly up-regulated genes and 1834 significantly down-regulated genes (Harms et al. 2014). Comparison of the two data sets revealed an overlap of 269 up-regulated genes but only 52 down-regulated genes (Fig. 2D). Assuming that the PRDM16 and LSD1 gene sets are completely independent, the $P$-value for the down-regulated gene sets to have an overlap of 52 or more is $\sim 0.24$, whereas the $P$-value for the up-regulated genes to have an overlap of 269 or more is $<0.00001$. Hence, this analysis indicates that there exists a much more significant overlap between the up-regulated genes in the LSD1-deficient and PRDM16-deficient BAT than those down-regulated by these manipulations in vivo. Moreover, we observed a strongly preferred presence for WAT-selective genes in the overlapping set of 269 genes but not the remaining 872 genes up-regulated in the LSD1-deficient BAT (Fig. 2D; Supplemental Fig. S2), suggesting that the common set of genes being repressed by PRDM16 and LSD1 is predominantly WAT-selective. We confirmed by quantitative PCR (qPCR) that the increased expression of WAT-selective genes occurs only in the homozygous but not the heterozygous LSD1 knockout BAT, suggesting that this is a phenotype specific to LSD1 ablation but not to the presence of the floxed allele or the CRE recombinase (Fig. 2E). Unlike in vitro experiments, we observed no reduction in the BAT-selective genes ucp1 and cidea in the LSD1-deficient BAT (Fig. $2 F)$. These results from global expression analyses are consistent with the effects of pharmacological inhibition or genetic ablation of LSD1 on gene expression in brown adipocytes differentiated in vitro and emphasize again that the corepressor function of LSD1 and PRDM16 is strongly correlated, whereas their abilities to activate gene transcription are largely independent.

\section{LSD1 and PRDM16 repression of WAT-selective genes correlates with the demethylation of $\mathrm{H} 3 \mathrm{~K} 4$}

We sought to determine whether the LSD1-mediated and PRDM16-mediated repression of WAT-selective genes reflected a direct action of these proteins; thus, we performed chromatin immunoprecipitation (ChIP) sequencing (ChIP-seq) of LSD1 and PRDM16 to identity their genomic binding sites. We identified 22,274 peaks in LSD1 ChIP-seq and 22,537 peaks in PRDM16 ChIP-seq (Fig. 3A). There are $9.7 \%$ of PRDM16 and $19.5 \%$ of LSD1 peaks located in the promoter region of target genes, both of which are significantly enriched relative to the genomic level (2.7\%) (Fig. 3A). There is a significant overlap of 11,843 between the two sets of peaks, marking sites of co-occupancy by LSD1 and PRDM16. We observed highly enriched motifs of the transcription factor CCAAT/enhancer-binding protein $\alpha(\mathrm{C} / \mathrm{EBPa})$ and the early B-cell factor (EBF) present in both the PRDM16 and LSD1 peaks (Fig. 3B). Indeed, we detected C/EBPa and EBF3 by mass spectrometry in the purified endogenous PRDM16 complex (Supplemental Table S2), suggesting that C/EBPa or EBF3 may be responsible for recruiting the PRDM16/ LSD1 complex to certain genomic loci. In addition, we observed the motif of transcription factor RE-1 silencing factor (REST) and SIX 5 to be highly enriched in the LSD1 but not the PRDM16 peaks (Fig. 3B), suggesting that, apart from interacting with $\mathrm{C} / \mathrm{EBPa}$ and EBF3, LSD1 may also associate with additional transcription factors and target to other genomic loci independently.

The genomic distribution of the PRDM16 and LSD1 peaks relative to their target genes of regulation was then analyzed. Among the common set of 269 genes that are up-regulated in both the PRDM16-deficient and LSD1-deficient BAT, 51 (19\%) genes have colocalized PRDM16 and LSD1 peaks in the promoter-proximal region, suggesting that these genes may be direct targets of repression by PRDM16 and LSD1. For example, we observed colocalization of PRDM16 and LSD1 in the promoter-proximal regions of WAT-selective genes such as retn and agt (Fig. 3C). This is likely an underestimation because the contribution from occupancy of distal enhancers by PRDM16 and LSD1 is not considered. Importantly, we observed significantly increased local levels of H3K4me1 or H3K4me2 near the promoter-proximal regions of WAT-selective genes that are targets of repression by PRDM16 and LSD1 in either PRDM16-deficient or LSD1-deficient BAT, such as retn and agt (Fig. 3C), further supporting the idea that repression of these genes is a direct consequence of local demethylation mediated by LSD1. Interestingly, loss of PRDM16 results in a much more extensive increase in the local H3K4me1 and $\mathrm{H} 3 \mathrm{~K} 4 \mathrm{me} 2$ level near the agt gene than what is observed with the LSD1-deficient BAT (Fig. 3C), which is consistent with the fact that agt is up-regulated by nearly 20fold in the PRDM16-deficient BAT but only twofold in the LSD1-deficient BAT. This suggests that additional mechanisms not involving LSD1 may be used to regulate histone methylation levels near PRDM16-binding sites.

\section{AdLKO mice have increased fat mass and reduced energy expenditure}

We then characterized the metabolic phenotype of the AdLKO mice. Strikingly, even on a chow diet, the AdLKO mice have significantly increased body weights relative to the control (1sd1 $1^{\operatorname{lox} P / \operatorname{lox} P}$ ) (Fig. 4A). The weight divergence is significant as early as 4 wk of age (Fig. 4A). Magnetic resonance imaging (MRI)-based body composition analysis revealed that the majority of the body mass difference between the AdLKO and control animals on a chow diet is coming from fat, whereas the lean mass of the two types of mice is comparable (Fig. 4B). The weights of the interscapular brown adipose depot, the inguinal subcutaneous white adipose depot, and the epididymal white adipose depot of the AdLKO mice increased relative to the control by $120 \%, 40 \%$, and $15 \%$ (Fig. 4C). By gross appearance, the AdLKO BAT looks larger and paler (Fig. 4D). This phenotype cannot be a consequence of CRE recombinase expression alone in the BAT, as the heterozygous BAT 
A

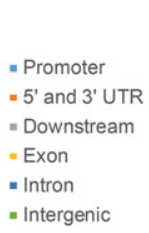

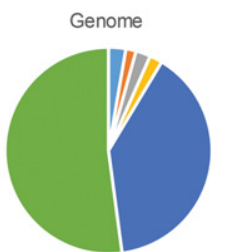
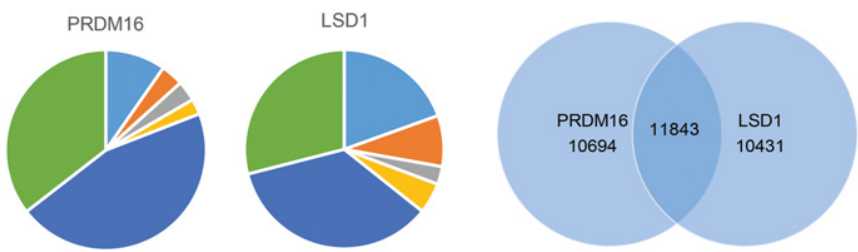

B

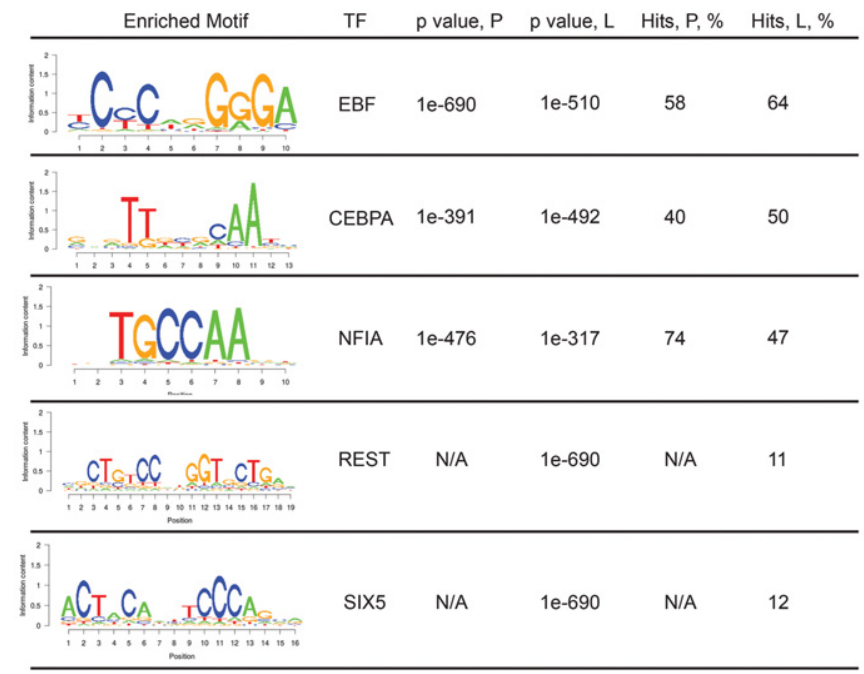

C

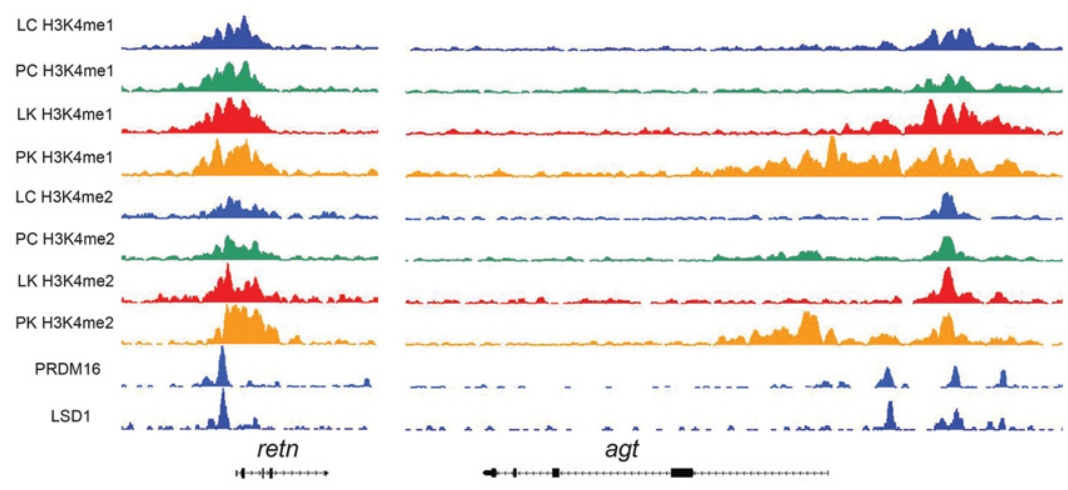

Figure 3. LSD1 and PRDM16 co-occupy loci near white-selective genes and regulate local histone methylation levels. (A) Genomic distribution of peaks identified in PRDM16 and LSD1 ChIP-seq. (B) Transcription factor motifs enriched in PRDM16 and LSD1 ChIP-seq peaks. (P) PRDM16 ChIP; (L) LSD1 ChIP. (C) H3K4me1 and H3K4me2 levels at the retn and agt loci in control and PRDM16-deficient or LSD1-deficient BAT. (LC) LSD1 floxed BAT; (LK) LSD1-deficient BAT; (PC) PRDM16 floxed BAT; (PK) PRDM16-deficient BAT.

$\left(1 s d 1^{l o x P /+} ;{ }^{2} e^{+}\right)$is indistinguishable from the control (Fig. 4D). Histological analysis of the AdLKO BAT and the inguinal WAT revealed a rather massive lipid accumulation relative to the control, whereas the AdLKO epididymal WAT was similar to the control (Fig. 4E). The histology results are consistent with the relative differences in gross tissue weights of different depots (Fig. 4C). At the whole-body level, the AdLKO mice have significantly reduced oxygen consumption and carbon dioxide production rates relative to the controls, with unaltered food intake and physical activity (Fig. 4F; Supplemental Fig. S3A,B). We also challenged the mice with a high-fat diet. Compared with the control mice, the AdLKO mice gained weight more rapidly (Supplemental Fig. S3C), showed reduced energy expenditure rate but unaltered food intake and movement (Supplemental Fig. S3D-F), and were less glucose-tolerant (Supplemental Fig. S3G). Because the BAT is highly active at performing FAO and thermogenesis, the most straightforward explanation for these phenotypes on the chow or high-fat diet is that ablation of LSD1 from the BAT results in a defect in FAO and thermogenesis, which in turn leads to lipid accumulation in all adipose depots and a reduction in whole-body energy expenditure. It is worth noting that mice rendered deficient for PRDM16 alone in the BAT do not display metabolic phenotypes of a similar magnitude at this young age 
A

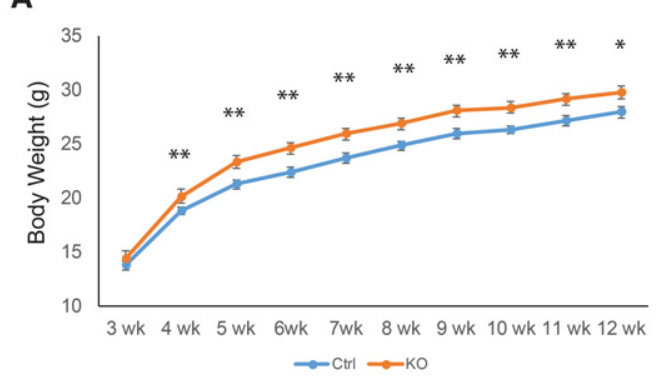

B
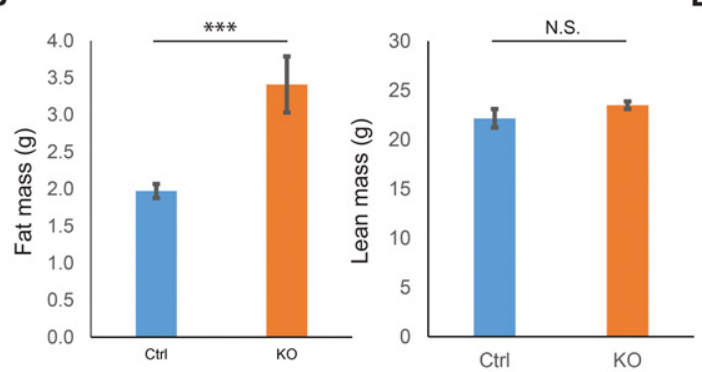

C

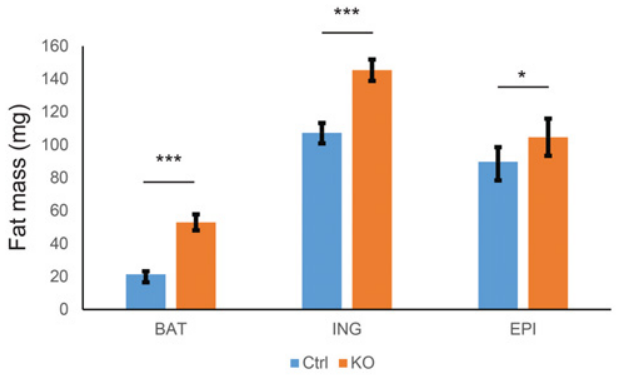

D

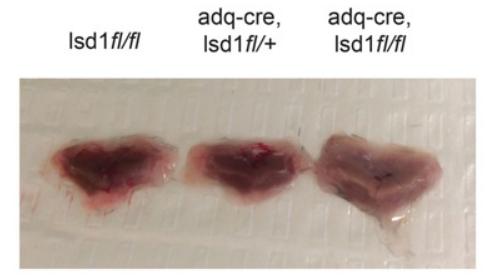

E

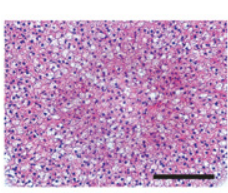

Ctrl BAT

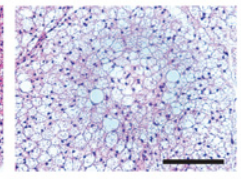

KO BAT

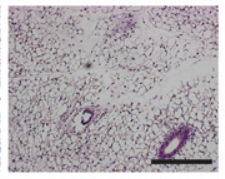

Ctrl ING

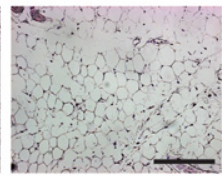

KO ING

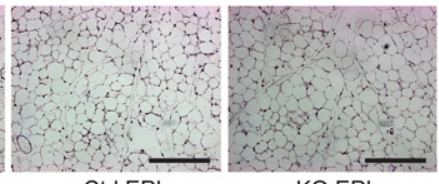

Ctrl EPI

KO EPI

$\mathbf{F}$
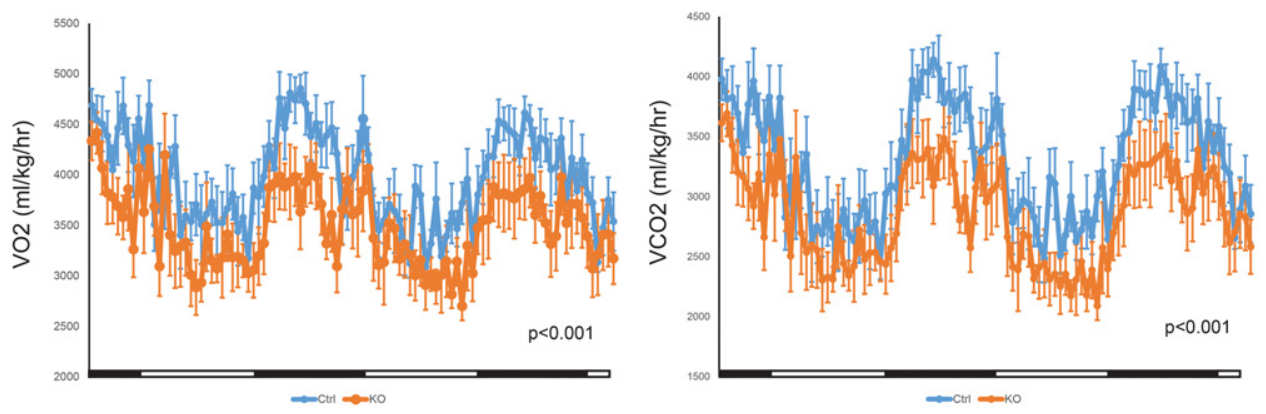

Figure 4. AdLKO mice display an obese phenotype. $(A)$ Body weight curves of male control and AdLKO mice on a chow diet. Mean \pm SEM. $n=15 .\left(^{*}\right) P<0.05 ;\left({ }^{* *}\right) P<0.01$. (B) Body composition of male control and AdLKO mice on a chow diet. Mean \pm SEM. $n=15$. $\left({ }^{* * *}\right)$ $P<0.001$. (C) Mass of individual fat depots of male control and AdLKO mice. Mean \pm SEM. $n=10 .\left({ }^{*}\right) P<0.05$; $\left(^{* * *}\right) P<0.001$. $(D)$ Gross appearance of BAT from control, heterozygous, and homozygous LSD1 knockout mice. $(E)$ Histology of BAT from control and AdLKO mice. Bar, $200 \mu \mathrm{m} .(F)$ Whole-body energy expenditure of control and AdLKO mice. Mean \pm SEM. $n=8 ; P<0.001$, two-way ANOVA. All mice were 8 wk old unless otherwise specified.

(Harms et al. 2014), suggesting that the phenotype of the AdLKO mice likely reflects regulatory roles of LSD1 in the BAT that are independent from Prdm16.

\section{AdLKO brown adipocytes have a blunted response to norepinephrine}

To assess whether the $\mathrm{FAO} /$ thermogenesis capacity is compromised in the AdLKO BAT, we measured the respiration rates of freshly isolated mature brown adipocytes from the AdLKO and control mice with a Clark-type oxygen electrode in the absence or presence of norepinephrine. Norepinephrine induces lipolysis in brown adipocytes, which both provides fuels for FAO and directly activates UCP1. Previous studies have established that the norepinephrine-induced mature brown adipocyte respiration is fully dependent on UCP1, and thus this assay offers a good estimate of BAT thermogenesis (Anita et al. 2000). Indeed, norepinephrine strongly stimulated respiration of the control cells, whereas this effect was 
blunted in the AdLKO cells, indicating a reduced thermogenic capacity (Fig. 5A). The dampened response to norepinephrine is unlikely to be due to weaker adrenergic receptor signaling, as the AdLKO cells released more glycerol in response to norepinephrine (Supplemental Fig. S4A). We also treated cells with oleate, which bypasses adrenergic signaling-driven lipolysis and directly stimulates brown adipocyte respiration in a fully UCP1-dependent manner (Anita et al. 2000). Again, the AdLKO brown adipocytes displayed a blunted response to oleate relative to the control (Fig. 5B). These results indicate at the cellular level that there is a defect in the FAO/thermogenic capacity of the AdLKO BAT, which probably lies downstream from the adrenergic signaling pathway.

Another possibility that may contribute to the lipid accumulation phenotype of the AdLKO BAT is increased lipogenesis. To measure lipogenesis, we incubated freshly isolated control or LSD1-deficient brown adipocytes with ${ }^{14} \mathrm{C}$-labeled glucose in the presence or absence of insulin and measured the amount of radioactivity incorporated into the triglyceride fraction extracted with organic solvents. The results showed that the LSD1deficient brown adipocytes converted a lower amount of glucose into triglyceride in both the presence and absence of insulin relative to the control (Supplemental Fig. S4B), which suggested that increased lipogenesis cannot be the reason accounting for the AdLKO BAT phenotype.

\section{AdLKO BAT mitochondria have reduced levels of FAO/oxidative phosphorylation proteins and slower respiration rates on fatty acid substrates}

To understand the molecular basis of the FAO/thermogenesis defect of the AdLKO BAT, we performed mass spectrometry-based proteomic analysis of the AdLKO and the control BAT. We were able to quantitate a total of 5704 proteins and identified 581 significantly up-regulated and 303 significantly down-regulated proteins in the AdLKO BAT relative to the control (Fig. 6A). A complete list of the proteins identified and quantitated is in Supplemental Table S1. We then performed gene set enrichment analysis (GSEA) on the proteins that are significantly different between the AdLKO and the control. Gene sets including mitochondrial oxidative phosphorylation (62), mitochondrial respiratory chain (18), and FAO (11) are found to be strongly enriched in the downregulated but not the up-regulated pool (Fig. 6B). When we plotted the position of each of the 200 genes in the oxidative phosphorylation set, the result clearly shows clustering of genes in the down-regulated region (42) relative to the up-regulated region (5) (Fig. 6C). This strongly argues that the enrichment of the oxidative phosphorylation set proteins in the down-regulated pool is not simply a consequence of the most abundant BAT proteins being detected by mass spectrometry but indeed reflects a phenotype specific to LSD1 ablation.

A closer inspection of the down-regulated proteins revealed multiple enzymes in the $\beta$-oxidation pathway and many components of the electron transport chain (ETC) (Supplemental Table S3). Down-regulation of such proteins suggests that the decreased respiration rates observed with isolated brown adipocytes from the AdLKO mice may be attributable to reduced mitochondrial FAO and ETC capacity. To test this idea, we measured the respiration rates of mitochondria isolated from the AdLKO and the control BAT with a Clark-type oxygen electrode. With palmitoyl-carnitine/malate as substrates, the AdLKO BAT mitochondria displayed a significantly lower respiration rate than the control (Fig. 6D). It is notable

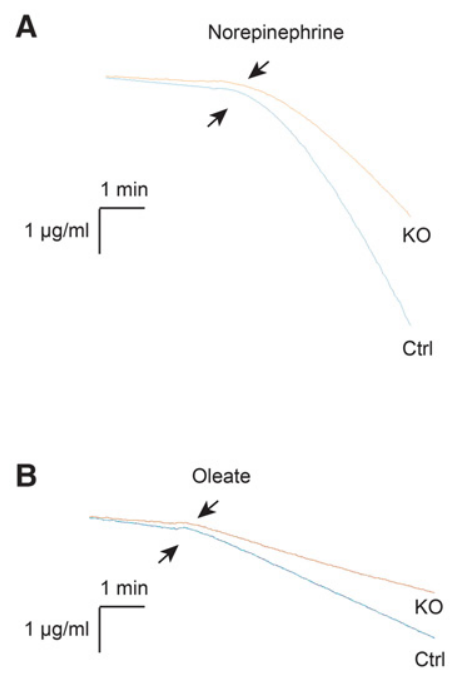

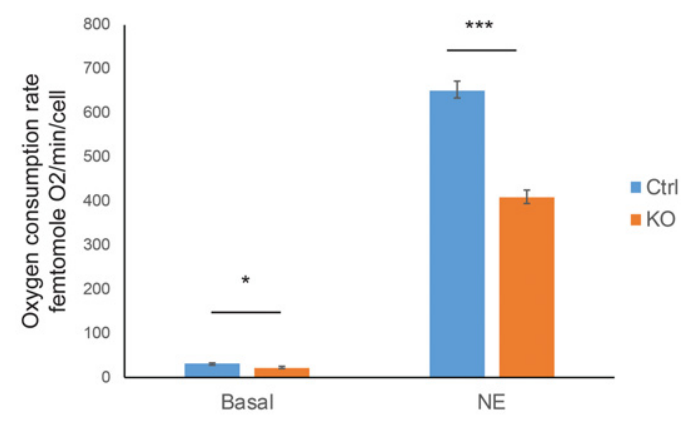

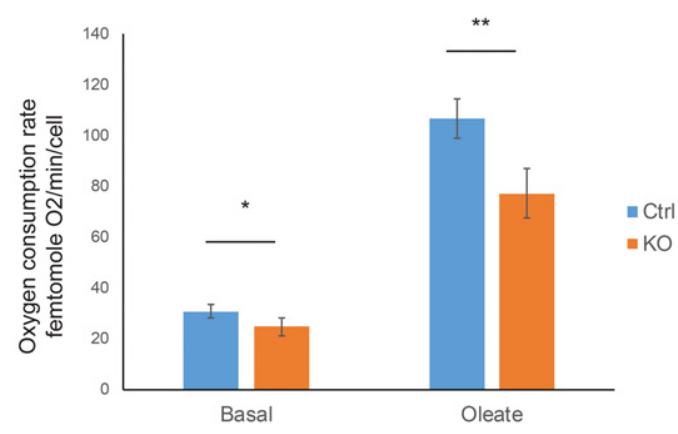

Figure 5. LSD1-deficient brown adipocytes have reduced respiration rates. $(A)$ Oxygen consumption rates of freshly isolated control and LSD1-deficient brown adipocytes in response to norepinephrine. Mean \pm SEM. $n=5$. $\left(^{*}\right) P<0.05$; $\left(^{* * *}\right) P<0.001$. A pair of representative raw traces are shown. (B) Oxygen consumption curves of freshly isolated control and LSD1-deficient brown adipocytes in response to oleate. Mean \pm SEM. $n=5$. $\left(^{*}\right) P<0.05$; $\left(^{* *}\right) P<0.01$. A pair of representative raw traces are shown. 
A

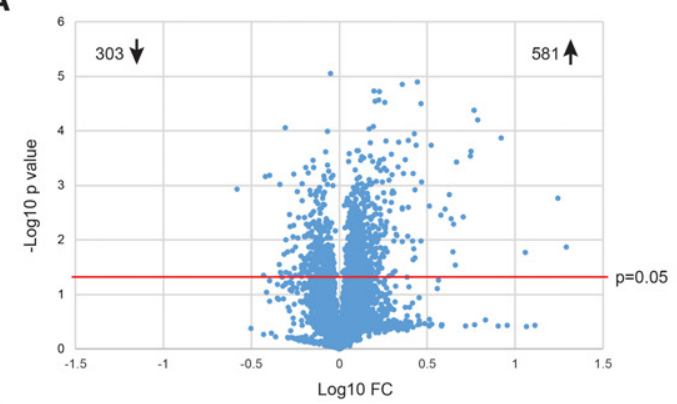

B
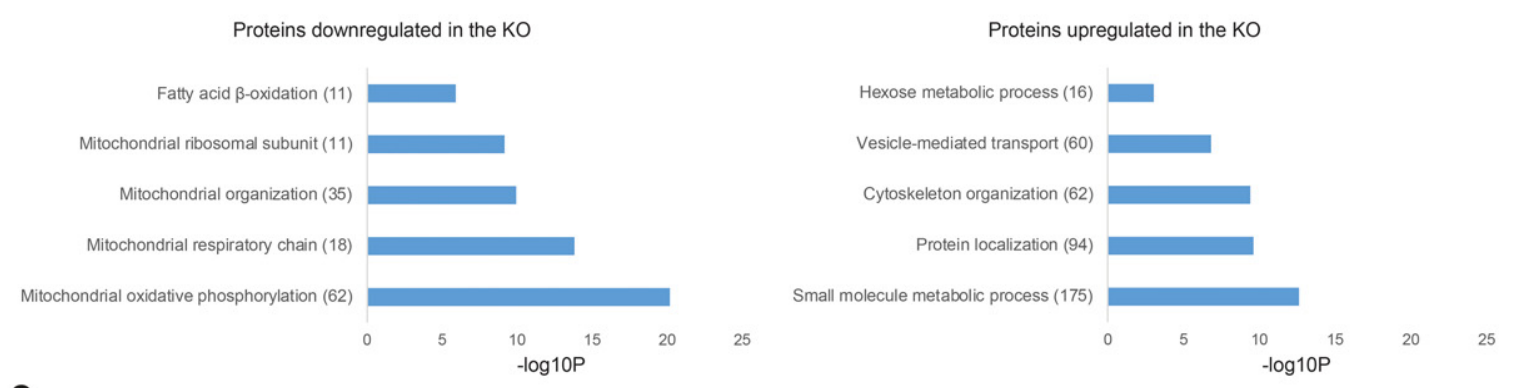

C

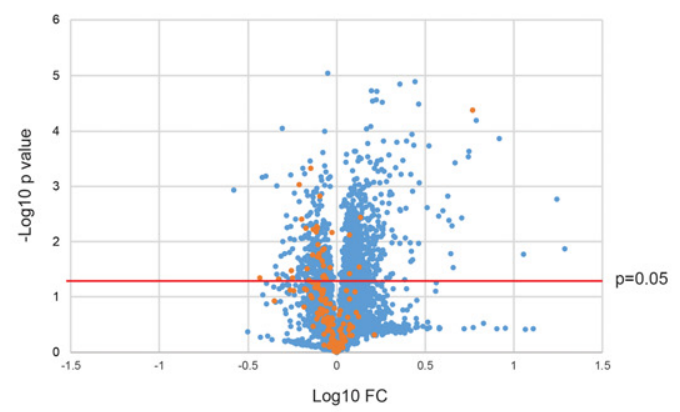

D
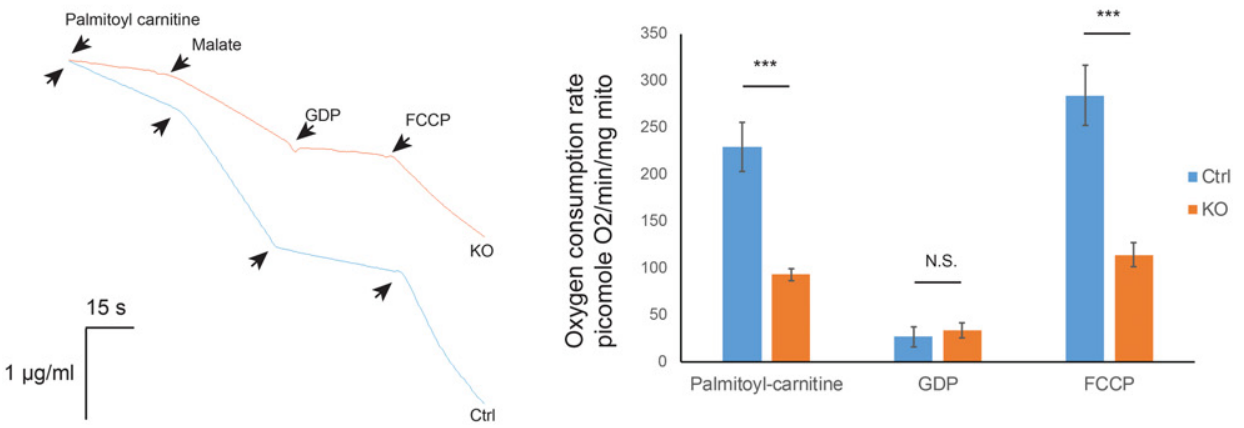

Figure 6. Mitochondria from LSD1-deficient BAT have reduced FAO capacity. (A) Volcano plot of mass spectrometry-based proteomics of the control and LSD1-deficient BAT. (B) GSEA of proteins that are significantly different between the control and LSD1-deficient BAT. $(C)$ Distribution of 200 proteins of the oxidative phosphorylation set in the volcano plot shown in $A$. (D) Oxygen consumption curves of mitochondria isolated from control and LSD1-deficient BAT with palmitoyl-cartinine as the substrate. (FCCP) Carbonyl cyanide-4-(trifluoromethoxy/phenylhydrazone. Mean \pm SEM. $n=5 .(* * *) P<0.001$. A pair of representative raw traces are shown.

that, although the magnitude of down-regulation of each individual component in the FAO and ETC pathway is modest, the overall rate of mitochondrial respiration when palmitoyl-carnitine is used as the substrate is reduced substantially $(\sim 50 \%)$, possibly reflecting a multipli- cative effect of reduced catalytic capacity at different steps (Spiegelman and Heinrich 2004). Taken together, these results strongly suggest that the whole-body metabolic phenotype of decreased energy expenditure rate and increased body fat accumulation of the AdLKO mice is likely at least 
in part due to reductions in the levels of mitochondrial proteins involved in the FAO and ETC pathway.

\section{Deletion of HSD11B1 results in a substantial rescue of the AdLKO BAT phenotype}

We sought to understand the mechanistic links between the ablation of LSD1 and the mitochondrial respiration defect of brown adipocytes. Because LSD1 is best known as a corepressor, we reasoned that the AdLKO BAT defect might be a consequence of derepression of certain critical LSD1 target genes. We therefore examined genes that are both abundantly expressed and strongly up-regulated in the AdLKO BAT relative to the control; our attention turned to the GC-activating enzyme HSD11B1, observed to be elevated in both the RNA-seq and proteomics data sets (Supplemental Table S1). We confirmed the up-regulation of HSD11B1 in the AdLKO BAT by qPCR (Fig. 7A). Importantly, HSD11B1 expression decreases modestly in the PRDM16-deficient BAT (Supplemental Fig. S5A), again highlighting that the LSD1-mediated repression of HSD11B1 reflects an independent activity from PRDM16.

HSD11B1 is responsible for generating the biologically active form of GC hormones (corticosterone in rodents) from the inert form (11-dehydrocorticosterone [11-DHC] in rodents) in various tissues, and GC excess is known to induce obesity in mammals (Seckl and Walker 2001). Interestingly, adipose-specific overexpression of HSD11B1 creates a metabolic phenotype similar to what we observed with the AdLKO mice (Masuzaki et al. 2001). We therefore tested the hypothesis that HSD11B1 is a direct target of repression by LSD1 and that the derepression of HSD11B1 followed by loss of LSD1 leads to BAT dysfunction. We observed increased levels of HSD11B1 mRNA in primary brown adipocytes differentiated in vitro under conditions of either pharmacological inhibition or genetic ablation of LSD1, suggesting that the elevated expression of HSD11B1 is a cell-autonomous effect of LSD1 inactivation (Supplemental Fig. S5B,C). LSD1 ChIP-seq revealed clear binding at the promoter region of hsd11b1, and the local level of H3K4me2 increases significantly in the LSD1-deficient BAT relative to the control (Fig. 7B), which provide evidence of direct repression. To obtain functional evidence that the up-regulation of HSD11B1 is responsible for the AdLKO BAT dysfunction, we crossed the AdLKO line into a previously reported global hsd11b1 knockout line to construct a double-knockout line (Semjonous et al. 2011). The deletion of HSD11B1 was confirmed by both Western blot (Fig. 7C) and HSD11B1 oxo-reductase activity assay (reduction of 11-DHC to corticosterone) conducted with minced BAT (Fig. 7D). We observed no difference in the serum corticosterone levels between mice of various genotypes (Supplemental Fig. S5D), suggesting that the elevated GC activation may be restricted to the adipose tissue. This same phenomenon was also observed with the adipose-specific HSD11B1 transgenic mouse (Masuzaki et al. 2001). On a chow diet, the morphology and triglyceride content of the hsd11b1 knockout (HKO) BAT are indistinguishable from the control, whereas the double-knockout BAT showed significant reduction in the triglyceride content relative to the AdLKO (Fig. 7E). This strongly suggests that the increased expression of HSD11B1 is indeed an important factor contributing to lipid accumulation and functional impairment of the AdLKO BAT. In line with this, mitochondria isolated from the double-knockout BAT displayed significantly higher respiration rates with palmitoyl-carnitine/malate as substrates than the AdLKO (Fig. 7F). Finally, at the whole-body level, the double-knockout mice were found to have higher energy expenditure rates than the AdLKO (Fig. 7G). Taken together, this evidence strongly supports that the upregulation of $\mathrm{HSD} 11 \mathrm{~B} 1$ is responsible for a substantial fraction of the BAT dysfunction observed in the AdLKO mice.

\section{Discussion}

In this study, we show that the repressive histone demethylase LSD1 physically interacts with and colocalizes with PRDM16 to genomic loci near WAT-selective genes that are targets of repression by PRDM16 in brown adipocytes. Loss of either LSD1 or PRDM16 results in up-regulation of local levels of $\mathrm{H} 3 \mathrm{~K} 4 \mathrm{me} 1$ and $\mathrm{H} 3 \mathrm{~K} 4 \mathrm{me} 2$ and increased expression of these WAT-selective genes in brown adipocytes. This strongly suggests that LSD1-mediated demethylation of $\mathrm{H} 3 \mathrm{~K} 4$ is a critical mechanism used by PRDM16 to achieve repression of WAT-selective genes in brown adipocytes. Ablation of LSD1 also causes impaired mitochondrial FAO/thermogenesis activity, at least in part due to elevated expression of HSD11B1, a direct target of repression by LSD1. Our studies thus provide insights into how epigenetic mechanisms regulate metabolic activity of adipocytes.

Previous studies also showed that the BAT and the skeletal muscle share a common developmental origin, as cells from both types of tissue are derived from a population of $\mathrm{myf5}^{+}$progenitors. Moreover, PRDM16 seems to repress the myogenic program while promoting adipogenesis during BAT development (Seale et al. 2008). Repression of the myogenic program is carried out by EHMT1, a repressive histone $\mathrm{H} 3 \mathrm{~K} 9$ methyltransferase copurified with PRDM16 (Ohno et al. 2013). Interestingly, most of the WAT-selective genes that are up-regulated in both the PRDM16-deficient and LSD1-deficient BAT do not change significantly in the EHMT1-deficient BAT, suggesting that EHMT1 selectively targets at the myogenic but not the WAT-selective genes (Ohno et al. 2013). On the other hand, we did not observe any up-regulation of myogenic genes in LSD1-deficient BAT (data not shown). It is therefore likely that LSD1 is specialized to repress WAT-selective genes but not myogenic genes together with PRDM16; thus, LSD1 mediates a distinct arm of the repressor function of PRDM16 from EHMT1. This division of labor is further supported by the observation that EHMT1 and CTBP1 interact with different fragments of PRDM16 (Ohno et al. 2013). Further study is required to clarify whether the PRDM16/EHMT1 and PRDM16/ LSD1 represent distinct complexes and whether the repression of WAT-selective or myogenic genes is mediated by distinct sequence-specific transcription factors. 
Zeng et al.

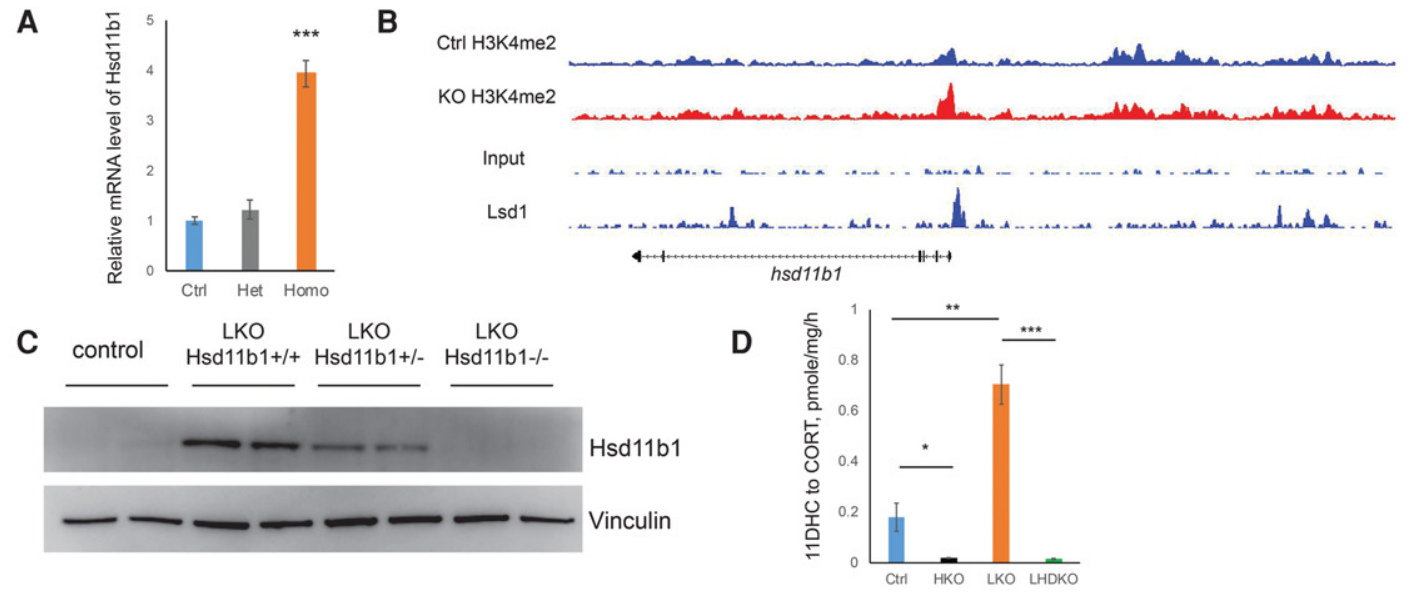

E
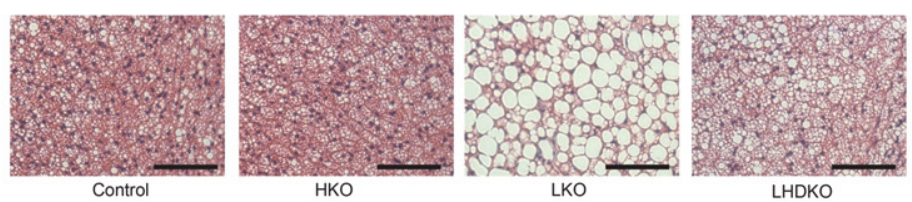

Triglyceride content mg/pad

F Palmitoyl-carnitine

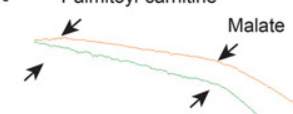

$1 \mu \mathrm{g} / \mathrm{ml}$

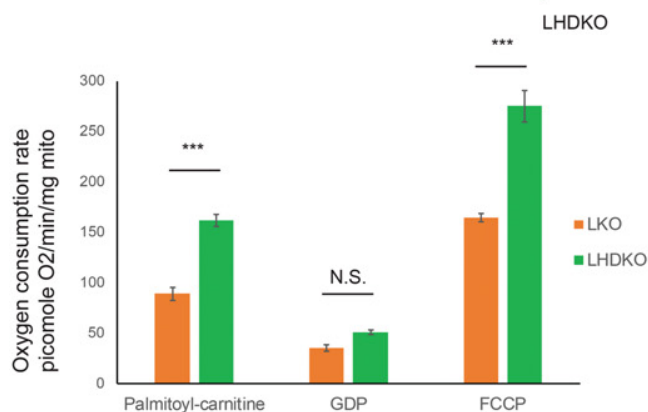

G
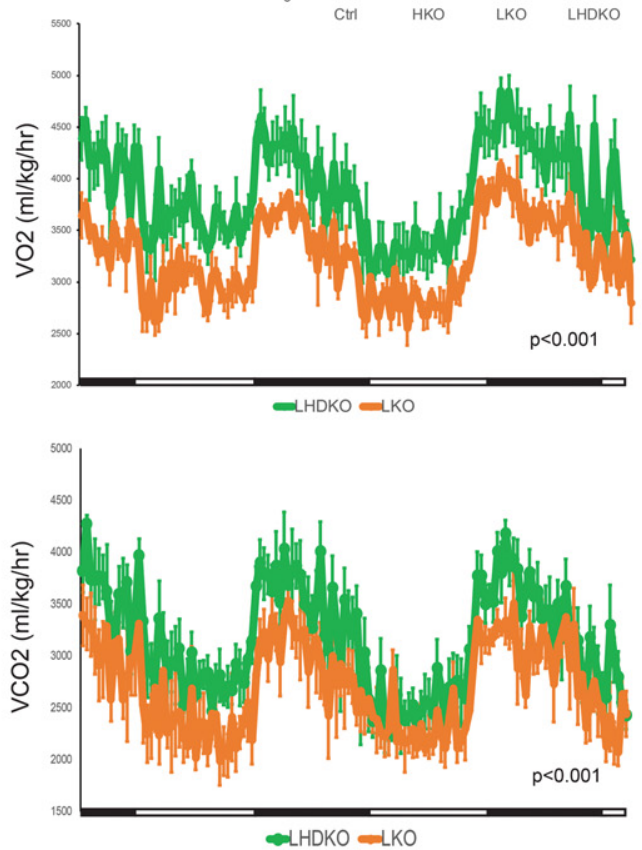

Figure 7. Simultaneous deletion of HSD11B1 substantially rescues the metabolic phenotype of the AdLKO mice. $(A)$ mRNA levels of HSD11B1 in the control and LSD1-deficient BAT. (AdLHKO) Heterozygous LSD1 knockout. Mean \pm SEM. $n=5$. $(* * *) P<0.001$. $(B)$ H3K4me2 levels near the HSD11B1 promoter region in the control and LSD1-deficient BAT. (C) Western blot for HSD11B1 in the control, LSD1 single-knockout, and LSD1/HSD11B1 double-knockout BAT. (D) HSD11B1 activity assay of control, HSD11B1 single-knockout (HKO), LSD1 single-knockout (LKO), and LSD1/HSD11B1 double-knockout (LHDKO) BAT. Mean \pm SEM. $n=5 .\left({ }^{*}\right) P<0.05$; $\left(^{* *}\right) P<0.01$; $\left(^{* * *}\right) P<0.001$. (E) Histology and triglyceride contents of the control, HSD11B1 single-knockout (HKO), LSD1 single-knockout (LKO), and LSD1/HSD11B1 double-knockout (LHDKO) BAT. Bar, $200 \mu \mathrm{m}$. $(F)$ Oxygen consumption rates of mitochondria isolated from LSD1 single-knockout (LKO) and LSD1/HSD11B1 double-knockout (LHDKO) BAT with palmitoyl-cartinine as the substrate. Mean \pm SEM. $n$ $\left.=5 .{ }^{* * *}\right) P<0.001$. A pair of representative raw traces are shown. $(G)$ Whole-body energy expenditure of LSD1 single-knockout (LKO) and LSD1/HSD11B1 double-knockout (LHDKO) mice. $P<0.001$, two-way ANOVA. All mice were 8 wk old.

Besides WAT-selective genes that are common repression targets of PRDM16 and LSD1, we also observed cooccupancy by PRDM16 and LSD1 at loci near some (but not all) BAT-selective genes that are targets of activation by PRDM16. Ablation of LSD1 has no effect on the levels of $\mathrm{H} 3 \mathrm{~K} 4 \mathrm{mel}$ and $\mathrm{H} 3 \mathrm{~K} 4 \mathrm{me} 2$ at those loci. These 
observations suggest that there is a mechanism that is preventing LSD1 from demethylating and inducing repression of those BAT-selective genes even if LSD1 is brought into close proximity together with PRDM16. Two reports showed that PRDM16 activates transcription of BAT-selective genes by recruiting MED1, a component of the mediator complex, to enhancer regions nearby (Harms et al. 2015; Iida et al. 2015). It is possible that the corecruitment of MED1 or additional members of the mediator complex by PRDM16 alters the enzymatic activity or substrate accessibility of LSD1 to render it nonoperative at loci near BAT-selective genes. This idea can be tested by replacing endogenous PRDM16 with a mutant that fails to interact with MED1 but still interacts with LSD1. This would predict that LSD1 may now demethylate and repress BAT-selective genes as well. Meanwhile, we also observed PRDM16 ChIP-seq peaks near BAT-selective genes that show no or a very low level of LSD1 binding, suggesting that there may exist PRDM16 complexes devoid of corepressors. These may be considered to be specialized in transcriptional activation. The composition of these potentially distinct PRDM16 complexes, especially the nature of sequence-specific transcription factors contained in each complex, deserves further investigation.

In two previous studies, Duteil et al. (2014) found that transgenic overexpression of LSD1 promotes expression of mitochondrial and thermogenic genes in the WAT, whereas Hino et al. (2012) made the opposite observation. Neither group used an adipose-specific loss-of-function model. Although we focused on the BAT in the present study, we did observe increased expression of some mitochondrial and thermogenic genes in the subcutaneous but not the visceral WAT of the AdLKO mice, suggesting that LSD1 may be involved in repressing the thermogenic program in beige adipocytes. Because beige adipocytes undergo a rapid "whitening" process upon reacclimation to warmth after cold exposure, there may exist a beige-specific mechanism to repress the thermogenic program, with LSD1 being a part of it. Future study focusing on the role of LSD1 in beige adipocytes may provide important insights into how these cells silence the thermogenic program.

In addition to LSD1, we also identified several other known components of the CTBP corepressor complex bound to PRDM16, and it will be interesting to know whether they share similar functions with LSD1 in the BAT. Among them, the role of ZFP516 in BAT function and development has been studied (Dempersmier et al. 2015). Interestingly, the BAT from ZFP516-null embryos displays strongly elevated myogenic gene expression but reduced or insignificant changes in WAT-selective gene expression, whereas we observed no significant change in myogenic gene expression but increased WAT-selective gene expression. This is in apparent contradiction to the fact that ZFP516 and LSD1 appear to be in the same PRDM16 complex. Such a paradox may arise from the different genetic models being used; we used a conditional knockout, whereas Dempersmier et al. (2015) used a global knockout. Therefore, this could be explained by the dis- tinct roles of the ZFP516 and LSD1 at different stages of BAT development. It will be interesting to develop a conditional knockout model of ZFP516 to see whether that recapitulates the phenotype of the AdLKO mice.

Another function of ZFP516 reported by Dempersmier et al. (2015) is activating ucp 1 transcription via demethylating $\mathrm{H} 3 \mathrm{~K} 9$ at the ucp 1 proximal promoter region in the inguinal WAT. While we were preparing this article, the same group published another paper suggesting that LSD1 is responsible for the H3K9 demethylation activity associated with ZFP516 (Sambeat et al. 2016). They further showed that BAT-specific LSD1 knockout mice displayed an obese phenotype similar to what we observed with the AdLKO mice and suggested that the reduced UCP1 expression might be one of the underlying mechanisms. We did not observe any reduction in UCP1 expression at both the protein and the mRNA level in the AdLKO BAT, arguing that decreased UCP1 transcription cannot account for the AdLKO BAT dysfunction. Moreover, as mentioned above, we found ucp 1 and a panel of other mitochondrial and thermogenic genes to be up-regulated in the inguinal WAT of the AdLKO mice, suggesting that LSD1 is repressing instead of activating the thermogenic program in the inguinal WAT. Our observation that deletion of HSD11B1 results in substantial alleviation of the LSD1-deficient BAT dysfunction is consistent with previous studies indicating that GC excess impairs BAT thermogenesis and promotes obesity (Strack et al. 1995; Masuzaki et al. 2001). At this stage, we cannot completely exclude the possibility that deletion of HSD11B1 in tissues other than the BAT may contribute to the improved BAT function. Constructing an adipose-specific LSD1/HSD11B1 double-knockout line will provide a more definitive answer. Meanwhile, it is clear that the double-knockout BAT still accumulates a significantly higher amount of lipid than the control, suggesting that additional mechanisms other than HSD11B1 derepression contribute to the BAT defect. Further studies will be required to understand the intimate balance between distinct fat cell programs influenced by LSD1.

\section{Materials and methods}

\section{Antibodies}

PRDM16 antibody for immunoprecipitation, ChIP, and Western blot was from R\&D Systems (AF6295). LSD1 antibody for Western blot was from Abcam (ab17721), and LSD1 antibody for ChIP was from EMD Millipore (05-939). CTBP1 antibody was from Abcam (ab129181). H3K4me1 antibody was from Diagenode (C1540194). H3K4me2 antibody was from EMD Millipore (07030). HSD11B1 antibody was from Abcam (ab39364).

\section{Animals}

$L s d 1^{\text {flox }}$ and $h s d 11 b 1$ knockout mice were obtained from the original publishers. The adiponectin-Cre line was maintained in the Spiegelman laboratory. All strains were on the C57Bl6 background. All animal experiments were approved by the Dana Farber Cancer Institute Institutional Animal Care and Use Committee. 


\section{Adenovirus production and purification}

Adenoviral expression constructs were made with the ViraPower adenoviral expression system (ThermoFisher, K493000). Amplified adenoviruses were purified with the Fast Trap adenovirus purification and concentration kit (EMD Millipore, FTAV00003), and the titer was determined with the Adeno-X rapid titer kit (Clontech, 632250).

\section{Cell cultures}

The stromal vascular fraction containing primary brown preadipocytes was isolated as described previously (Harms et al. 2014). Cells were induced to differentiate after reaching confluency with medium containing $10 \%$ FBS, $0.5 \mathrm{mM}$ isobutylmethylxanthine, $125 \mu \mathrm{M}$ indomethacin, $5 \mu \mathrm{M}$ dexamethasone, $80 \mathrm{nM}$ insulin, and $1 \mathrm{nM}$ T3. For adenoviral infection, cells were incubated with the viruses on day 2 after the start of differentiation at a multiplicity of infection (MOI) of $\sim 1: 100$ overnight. For pharmacological inhibition of LSD1, cells were treated with S2101 (Calbiochem, 489477) from days 2 to 6 after the start of differentiation.

\section{Protein purification}

To purify endogenous PRDM16, PRDM16 antibody was covalently coupled to protein G Dynabeads (ThermoFisher, 10004D) following the manufacturer's instructions. Primary brown preadipocytes were isolated from 40 pups, split to 4010 $\mathrm{cm}$ plates, and induced to differentiate. On day 4 after differentiation, cells were scraped off the plates, and nuclear extract was prepared as described previously (Dignam et al. 1983). The nuclear extract was incubated with the antibody beads for $3 \mathrm{~h}$ at $4^{\circ} \mathrm{C}$ and washed extensively. Bound proteins were eluted with $0.1 \mathrm{M}$ glycine ( $\mathrm{pH} 2.5)$ and neutralized with Tris. To purify Flag-tagged PRDM16, anti-Flag M2 affinity gel (Sigma, A2220) was used. Bound proteins were eluted with $100 \mu \mathrm{g} / \mathrm{mL}$ 3xFlag peptide (Sigma, F4799).

\section{Mass spectrometry}

A detailed description of the mass spectrometry experimental procedure is in the Supplemental Material.

\section{ChIP-seq}

BAT from 10-15 mice (male, 8-10 wk old) were dissected and homogenized with a douncer. Paraformaldehyde was added to the homogenate to $1 \%$ final concentration. Homogenization was performed prior to cross-linking because it is difficult to collect mature brown adipocytes by centrifugation. The homogenate was then quenched with $125 \mathrm{mM}$ glycine, and the nuclei were collected by centrifugation. The nuclei were lysed with lysis buffer $1 \%$ SDS, $50 \mathrm{mM}$ Tris-HCl, $10 \mathrm{mM}$ EDTA at $\mathrm{pH}$ 8.0) and sonicated to yield an average size of DNA fragment of 150-200 base pairs (bp). The lysate was diluted 10 -fold with a dilution buffer $(0.01 \%$ SDS, $1.1 \%$ Triton X-100, 1.2 mM EDTA, 16.7 mM Tris-HCl, $167 \mathrm{mM}$ $\mathrm{NaCl}$ at $\mathrm{pH} 8.0$ ) and incubated with the desired antibody overnight. The protein-DNA complex was isolated with protein G Dynabeads, washed, and eluted off the beads. The eluent was treated with RNase A and proteinase $\mathrm{K}$ before reversing crosslinking. DNA was then harvested by ethanol precipitation, and a library was prepared with the NEBNext ChIP-seq library preparation master mix set for Illumina (New England Biolabs, E6240S). The library was sequenced on an Illumina Nextseq
500 sequencer, and raw reads were mapped with the mapping tool in the CLC Genomics Workbench software. Peaks were called with the model-based analysis for ChIP-seq (MACS) method, and the $P$-value threshold was set at 0.05 . Subsequent analyses, including genomic distribution, peak set overlap, and transcription factor motif enrichment, were performed with tools on the DFCI Galaxy Cistrome Web site.

\section{RNA-seq}

Total RNA was isolated from BAT with the Trizol reagent (ThermoFisher, 15596026). mRNA was purified with the Magenic mRNA isolation kit (New England Biolabs, S1550S), and a library was prepared with the NEBNext Ultra RNA library preparation kit for Illumina (New England Biolabs, E7530S). The library was sequenced on an Illumina Nextseq 500 sequencer. Raw reads were aligned using a STAR aligner (version STAR_2.4.2a), and raw gene counts were quantified using the quantMode GeneCounts flag. Differential expression testing was performed using Limma and DESeq2.

\section{Metabolic phenotyping}

All experiments were done with male mice. Energy expenditure was analyzed using a Comprehensive Lab Animal Monitoring System (Columbus Instruments). Fat and lean mass were measured by MRI.

\section{Respiration assay}

To measure mature brown adipocyte respiration, BAT was dissected, minced, and digested with Krebs Ringer bicarbonate-modified buffer (KRBMB; $120 \mathrm{mM} \mathrm{NaCl}, 5 \mathrm{mM} \mathrm{KCl}, 1 \mathrm{mM} \mathrm{MgCl}_{2}$, $1 \mathrm{mM} \mathrm{CaCl}_{2}, 0.4 \mathrm{mM} \mathrm{K}_{2} \mathrm{HPO}_{4}, 10 \mathrm{mM}$ glucose, $15 \mathrm{mM} \mathrm{NaHCO}_{3}$, $20 \mathrm{mM}$ HEPES at $\mathrm{pH} 7.4,4 \%$ fatty acid free BSA), $2 \mathrm{mg} / \mathrm{mL}$ collagenase $\mathrm{B}$ (Worthington, CLSAFB), and $1 \mathrm{mg} / \mathrm{mL}$ soybean trypsin inhibitor (Worthington, LS003570) for $30 \mathrm{~min}$ at $37^{\circ} \mathrm{C}$. Cells were washed three times with KRBMB and allowed to recover for at least $30 \mathrm{~min}$ at room temperature. An oxygen electrode (Strathkelvin Instrument, SI130) installed in a respiration chamber (StrathKelvin Instrument, MT200A) was used to measure oxygen consumption rate. Cells were counted by lysis followed by Trypan blue staining of the nuclei. For each run, 150,000 cells were suspended in $1 \mathrm{~mL}$ of $\mathrm{KRBMB}$ and added to the respiration chamber. Norepinephrine $(1 \mu \mathrm{M})$ or $3 \mathrm{mM}$ sodium oleate was injected into the chamber to stimulate respiration. To measure mitochondrial respiration, crude mitochondria were isolated from the BAT following a procedure described previously (Nedergaard and Cannon 1979). For each run, an amount of $200 \mu \mathrm{g}$ of protein was used. Palmitoyl-carnitine $(40 \mu \mathrm{M}), 5 \mathrm{mM}$ malate, $1 \mathrm{mM}$ GDP, and $1 \mu \mathrm{M}$ FCCP were sequentially injected into the chamber.

\section{HSD11B1 activity assay}

Freshly dissected BAT ( 10 mg) was minced and suspended in $2 \mathrm{~mL}$ of serum-free medium supplemented with or without $100 \mathrm{nM} 11-\mathrm{DHC}$ and incubated for $2 \mathrm{~h}$ at $37^{\circ} \mathrm{C}$. The supernatant was collected, and the corticosterone level was measured by ELISA (ab108821). Samples without 11-DHC were used for background subtraction. 


\section{Statistical analysis}

The Student's $t$-test was used for single comparisons. Two-way ANOVA with repeated measures was used for body weight and energy expenditure comparisons.

\section{Acknowledgments}

We thank Stuart Orkin and Marc Kerenyi for generously sharing the $1 s d 1^{f l o x}$ animals, Yang Shi and Benoît Laurent for providing the LSD1 constructs and experimental advice, Zachary Herbert and the Molecular Biology Core Facilities at Dana Farber Cancer Institute for sequencing studies, the Rodent Histology Core at Harvard Medical School for histology studies, and the Nikon Imaging Center at Harvard Medical School for immunofluorescence studies. This study was supported by National Institutes of Health grant DK31405 to B.M.S., and an American Heart Association post-doctoral fellowship to X.Z.

\section{References}

Anita M, Kerstin BEO, Magnus JF, Anders J, Jan N, Barbara C. 2000. Thermogenic responses in brown fat cells are fully UCP1-dependent UCP2 or UCP3 do not substitute for UCP1 in adrenergically or fatty acid-induced thermogenesis. I Biol Chem 275: 25073-25081.

Basen-Engquist K, Chang M. 2011. Obesity and cancer risk: recent review and evidence. Curr Oncol Rep 13: 71-76.

Cannon B, Nedergaard J. 2004. Brown adipose tissue: function and physiological significance. Physiol Rev 84: 277-359.

Cohen P, Levy J, Zhang Y, Frontini A, Kolodin D, Svensson K, Lo J, Zeng X, Ye L, Khandekar M, et al. 2014. Ablation of PRDM16 and beige fat causes metabolic dysfunction and subcutaneous to visceral adipose swtich. Cell 156: 304-316.

Dempersmier J, Sambeat A, Gulyaeva O, Paul SM, Hudak CSS, Raposo HF, Kwan H-Y, Kang C, Wong RH, Sul HS. 2015. Cold-inducible Zfp516 activates UCP1 transcription to promote browning of white fat and development of brown fat. Mol Cell 57: 235-246.

Després J-P, Lemieux I. 2006. Abdominal obesity and metabolic syndrome. Nature 444: 881-887.

Dignam JD, Lebovitz RM, Roeder RG. 1983. Accurate transcription initiation by RNA polymerase II in a soluble extract from isolated mammalian nuclei. Nucleic Acids Res 11: 1475-1489.

Dulloo AG, Miller DS. 1984. Energy balance following sympathetic denervation of brown adipose tissue. Can I Physiol Pharmacol 62: 235-240.

Duteil D, Metzger E, Willmann D, Karagianni P, Friedrichs N, Greschik H, Günther T, Buettner R, Talianidis I, Metzger D, et al. 2014. LSD1 promotes oxidative metabolism of white adipose tissue. Nat Commun 5: 4093.

Gaal LF, Mertens IL, Block CE. 2006. Mechanisms linking obesity with cardiovascular disease. Nature 444: 875-880.

Ghorbani M, Himms-Hagen J. 1997. Appearance of brown adipocytes in white adipose tissue during CL 316,243-induced reversal of obesity and diabetes in Zucker fa/fa rats. Int $J$ Obes Relat Metab Disord 21: 465-475.

Harms MJ, Ishibashi J, Wang W, Lim H-W, Goyama S, Sato T, Kurokawa M, Won K-J, Seale P. 2014. Prdm16 is required for the maintenance of brown adipocyte identity and function in adult mice. Cell Metab 19: 593-604.

Harms MJ, Lim H-W, Ho Y, Shapira SN, Ishibashi J, Rajakumari S, Steger DJ, Lazar MA, Won K-J, Seale P. 2015. PRDM16 binds
MED1 and controls chromatin architecture to determine a brown fat transcriptional program. Genes Dev 29: 298-307.

Hino S, Sakamoto A, Nagaoka K, Anan K, Wang Y, Mimasu S, Umehara T, Yokoyama S, Kosai K-i, Nakao M. 2012. FAD-dependent lysine-specific demethylase-1 regulates cellular energy expenditure. Nat Commun 3: 758.

Iida S, Chen W, Nakadai T, Ohkuma Y, Roeder RG. 2015. PRDM16 enhances nuclear receptor-dependent transcription of the brown fat-specific Ucp1 gene through interactions with Mediator subunit MED1. Genes Dev 29: 308-321.

Kahn SE, Hull RL, Utzschneider KM. 2006. Mechanisms linking obesity to insulin resistance and type 2 diabetes. Nature 444: 840-846.

Kajimura S, Seale P, Tomaru T, Erdjument-Bromage H, Cooper M, Ruas J, Chin S, Tempst P, Lazar M, Spiegelman B. 2008. Regulation of the brown and white fat gene programs through a PRDM16/CtBP transcriptional complex. Genes Dev 22: 1397-1409.

Kerenyi MA, Shao Z, Hsu Y-J, Guo G, Luc S, O’Brien K, Fujiwara Y, Peng C, Nguyen M, Orkin SH. 2013. Histone demethylase Lsd1 represses hematopoietic stem and progenitor cell signatures during blood cell maturation. Elife 2: e00633.

Loncar D. 1991. Convertible adipose tissue in mice. Cell Tissue Res 266: 149-161.

Long IZ, Svensson KJ, Tsai L, Zeng X, Roh HC, Kong X, Rao RR, Lou J, Lokurkar I, Baur W, et al. 2014. A smooth muscle-like origin for beige adipocytes. Cell Metab 19: 810-820.

Masuzaki H, Paterson J, Shinyama H, Morton NM, Mullins JJ, Seckl JR, Flier JS. 2001. A transgenic model of visceral obesity and the metabolic syndrome. Science (New York, N.Y.) 294: 2166-2170.

Matthew JH, Jeff I, Wenshan W, Hee-Woong WL, Susumu G, Tomohiko S, Mineo K, Kyoung-Jae JW, Patrick S. 2014. Prdm16 is required for the maintenance of brown adipocyte identity and function in adult mice. Cell Metab 19: 593-604.

Minokoshi Y, Saito M, Shimazu T. 1986. Metabolic and morphological alterations of brown adipose tissue after sympathetic denervation in rats. J Auton Nerv Syst 15: 197-204.

Nedergaard J, Cannon B. 1979. Overview-preparation and properties of mitochondria from different sources. Methods Enzymol 55: 3-28.

Ohno H, Shinoda K, Ohyama K, Sharp LZ, Kajimura S. 2013. EHMT1 controls brown adipose cell fate and thermogenesis through the PRDM16 complex. Nature 504: 163-167.

Sambeat A, Gulyaeva O, Dempersmier J, Tharp KM, Stahl A, Paul SM, Sul H. 2016. LSD1 interacts with Zfp516 to promote UCP1 transcription and brown fat program. Cell Rep 15: 2536-2549.

Seale P, Kajimura S, Yang W, Chin S, Rohas L, Uldry M, Tavernier G, Langin D, Spiegelman B. 2007. Transcriptional control of brown fat determination by PRDM16. Cell Metab 6: 38-54.

Seale P, Bjork B, Yang W, Kajimura S, Chin S, Kuang S, Scimè A, Devarakonda S, Conroe HM, Erdjument-Bromage H, et al. 2008. PRDM16 controls a brown fat/skeletal muscle switch. Nature 454: 961-967.

Seale P, Conroe H, Estall J, Kajimura S, Frontini A, Ishibashi J, Cohen P, Cinti S, Spiegelman B. 2011. Prdm16 determines the thermogenic program of subcutaneous white adipose tissue in mice. J Clin Invest 121: 96-105.

Seckl JR, Walker BR. 2001. Minireview: 11ß-hydroxysteroid dehydrogenase type 1- a tissue-specific amplifier of glucocorticoid action. Endocrinology 142: 1371-1376.

Semjonous NM, Sherlock M, Jeyasuria P, Parker KL, Walker EA, Stewart PM, Lavery GG. 2011. Hexose-6-phosphate dehydrogenase contributes to skeletal muscle homeostasis 
independent of $11 \beta$-hydroxysteroid dehydrogenase type 1 . Endocrinology 152: 93-102.

Shi Y, Sawada J-i, Sui G, Affar el B, Whetstine JR, Lan F, Ogawa H, Luke MP, Nakatani Y, Shi Y. 2003. Coordinated histone modifications mediated by a CtBP co-repressor complex. Nature 422: 735-738.

Shi Y, Lan F, Matson C, Mulligan P, Whetstine J, Cole P, Casero R, Shi Y. 2004. Histone demethylation mediated by the nuclear amine oxidase homolog LSD1. Cell 119: 941-953.
Spiegelman BM, Heinrich R. 2004. Biological control through regulated transcriptional coactivators. Cell 119: 157-167.

Strack AM, Bradbury MJ, Dallman MF. 1995. Corticosterone decreases nonshivering thermogenesis and increases lipid storage in brown adipose tissue. Am J Physiol 268: R183-R191.

Wu J, Boström P, Sparks L, Ye L, Choi J, Giang A-H, Khandekar M, Virtanen K, Nuutila P, Schaart G, et al. 2012. Beige adipocytes are a distinct type of thermogenic fat cell in mouse and human. Cell 150: 366-376. 


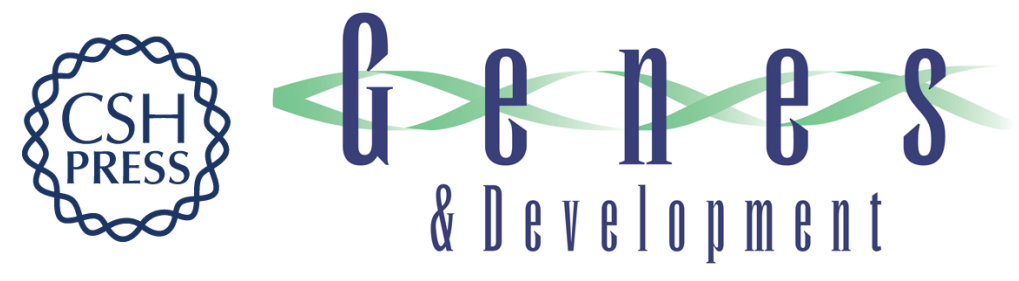

\section{Lysine-specific demethylase 1 promotes brown adipose tissue thermogenesis via repressing glucocorticoid activation}

Xing Zeng, Mark P. Jedrychowski, Yi Chen, et al.

Genes Dev. 2016, 30: originally published online August 26, 2016

Access the most recent version at doi:10.1101/gad.285312.116

\section{Supplemental http://genesdev.cshlp.org/content/suppl/2016/08/26/gad.285312.116.DC1 \\ Material}

Related Content

References

Creative

Commons

License

Email Alerting

Service
LSD1a pivotal epigenetic regulator of brown and beige fat differentiation and homeostasis

Jean Z. Lin and Stephen R. Farmer

Genes Dev. August , 2016 30: 1793-1795

This article cites 36 articles, 4 of which can be accessed free at:

http://genesdev.cshlp.org/content/30/16/1822.full.html\#ref-list-1

Articles cited in:

http://genesdev.cshlp.org/content/30/16/1822.full.html\#related-urls

This article is distributed exclusively by Cold Spring Harbor Laboratory Press for the first six months after the full-issue publication date (see

http://genesdev.cshlp.org/site/misc/terms.xhtml). After six months, it is available under a Creative Commons License (Attribution-NonCommercial 4.0 International), as described at http://creativecommons.org/licenses/by-nc/4.0/.

Receive free email alerts when new articles cite this article - sign up in the box at the top right corner of the article or click here.

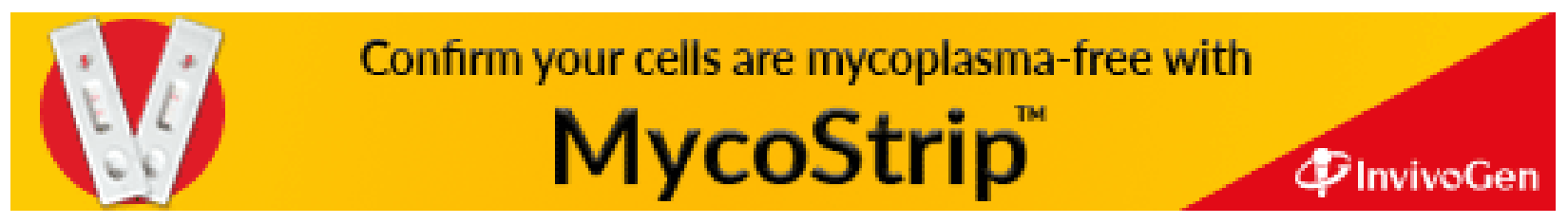

